Bull. Korean Math. Soc. 52 (2015), No. 5, pp. 1481-1487

http://dx.doi.org/10.4134/BKMS.2015.52.5.1481

\title{
SUPERCYCLICITY OF JOINT ISOMETRIES
}

\author{
Mohammad Ansari, Karim Hedayatian, Bahram Khani-Robati, \\ AND ABBAS MORADI
}

\begin{abstract}
Let $H$ be a separable complex Hilbert space. A commuting tuple $T=\left(T_{1}, \ldots, T_{n}\right)$ of bounded linear operators on $H$ is called a spherical isometry if $\sum_{i=1}^{n} T_{i}^{*} T_{i}=I$. The tuple $T$ is called a toral isometry if each $T_{i}$ is an isometry. In this paper, we show that for each $n \geq 1$ there is a supercyclic $n$-tuple of spherical isometries on $\mathbb{C}^{n}$ and there is no spherical or toral isometric tuple of operators on an infinite-dimensional Hilbert space.
\end{abstract}

\section{Introduction}

An $n$-tuple of operators is a finite sequence of length $n$ of commuting bounded linear operators $T_{1}, T_{2}, \ldots, T_{n}$ acting on a Hilbert space $H$. For an $n$-tuple $T=\left(T_{1}, T_{2}, \ldots, T_{n}\right)$, if there exists an element $x \in H$ such that $\operatorname{orb}(T, x)=$ $\left\{S x: S \in \mathcal{F}_{T}\right\}$ where $\mathcal{F}_{T}=\left\{T_{1}^{k_{1}} T_{2}^{k_{2}} \cdots T_{n}^{k_{n}}: k_{i} \geq 0, i=1,2, \ldots, n\right\}$, is dense in $H$ then $x$ is called a hypercyclic vector for $T$, and $T$ is said to be a hypercyclic $n$-tuple of operators. A vector $x \in H$ is called a supercyclic vector for $T$ if the set $\left\{\lambda S x: S \in \mathcal{F}_{T}, \lambda \in \mathbb{C}\right\}$ is dense in $H$, and $T$ is said to be a supercyclic $n$-tuple of operators. These definitions generalize the notions of hypercyclicity and supercyclicity of a single operator to a tuple of operators. Hypercyclicity and supercyclicity of tuples of operators have been investigated in $([3],[4],[6],[7])$. On the other hand, spherical isometries are a considerable part of tuples of operators. The authors in [5] proved that isometries on Hilbert spaces with dimension more than one are not supercyclic. Recently, this fact has been proved for $m$-isometric operators which are a generalization of isometric operators in some sense [2]. In this paper we see that spherical isometries are not supercyclic on infinite-dimensional Hilbert spaces. Let $A$ be a matrix we denote by $A^{T}$ and $\operatorname{det} A$ the transpose and the determinant of $A$ respectively.

In Section 2, we show that there is no supercyclic $n$-tuple of diagonalizable matrices on $\mathbb{C}^{n+1}$. The main result of this section is that there is a supercyclic

Received June 11, 2014; Revised March 2, 2015.

2010 Mathematics Subject Classification. 47A16.

Key words and phrases. supercyclicity, tuples, subnormal operators, spherical isometry, toral isometry. 
$p$-tuple of spherical isometries on $\mathbb{C}^{p}, p \geq 1$. Also, it is proved that there is no spherical or toral isometric tuple of operators on an infinite-dimensional Hilbert space.

\section{Supercyclicity of spherical isometries}

In [4] it is shown that for each $n \geq 1$, there exists a hypercyclic $(n+1)$-tuple of diagonal matrices on $\mathbb{C}^{n}$, and there is no hypercyclic $n$-tuple of diagonalizable matrices on $\mathbb{C}^{n}$. We give the following result which is a generalization of $[4$, Theorem 3.6]. The technique employed in the proof is due to N. Feldman.

Theorem 1. There is no supercyclic n-tuple of diagonalizable matrices on $\mathbb{C}^{n+1}$.

Proof. The proof is on the same lines as for the case $n=2$. We assume that there exists a supercyclic 2-tuple $(A, B)$ of diagonalizable matrices on $\mathbb{C}^{3}$. We can assume that the matrices $A$ and $B$ are diagonal thanks to simultaneously diagonalizability. Let

$$
A=\left[\begin{array}{ccc}
a_{1} & 0 & 0 \\
0 & a_{2} & 0 \\
0 & 0 & a_{3}
\end{array}\right], B=\left[\begin{array}{ccc}
b_{1} & 0 & 0 \\
0 & b_{2} & 0 \\
0 & 0 & b_{3}
\end{array}\right]
$$

and $\nu=\left[\begin{array}{l}\alpha \\ \beta \\ \gamma\end{array}\right]$ be a supercyclic vector for the 2-tuple $(A, B)$. Therefore, if

$$
E=\left\{\left[\begin{array}{l}
\lambda a_{1}^{n} b_{1}^{k} \alpha \\
\lambda a_{2}^{n} b_{2}^{k} \beta \\
\lambda a_{3}^{n} b_{3}^{k} \gamma
\end{array}\right]: \lambda \in \mathbb{C}, n, k \geq 0\right\}=\left\{\lambda A^{n} B^{k} \nu: \lambda \in \mathbb{C}, n, k \geq 0\right\},
$$

then $\bar{E}=\mathbb{C}^{3}$ which in turn implies that $\alpha, \beta, \gamma, a_{i}$ and $b_{i}$ for $i=1,2,3$ are nonzero. Since the 2-tuple $\left(\frac{1}{a_{1}} A, \frac{1}{b_{1}} B\right)$ is also supercyclic, we may assume that $a_{1}=b_{1}=1$. On the other hand, by applying the invertible matrix

$$
\left[\begin{array}{ccc}
\alpha^{-1} & 0 & 0 \\
0 & \beta^{-1} & 0 \\
0 & 0 & \gamma^{-1}
\end{array}\right]
$$

to the set $E$ we conclude that $\bar{F}=\mathbb{C}^{3}$ where

$$
F=\left\{\left[\begin{array}{c}
\lambda \\
\lambda a_{2}^{n} b_{2}^{k} \\
\lambda a_{3}^{n} b_{3}^{k}
\end{array}\right]: \lambda \in \mathbb{C}, n, k \geq 0\right\} .
$$

Now by applying the function $\log |z|$ to each coordinate of the set $F$ we have $\bar{G}=\mathbb{R}^{3}$ where

$$
G=\left\{\left[\begin{array}{c}
\log |\lambda| \\
n \log \left|a_{2}\right|+k \log \left|b_{2}\right|+\log |\lambda| \\
n \log \left|a_{3}\right|+k \log \left|b_{3}\right|+\log |\lambda|
\end{array}\right]: n, k \geq 0, \lambda \in \mathbb{C}\right\} .
$$


Let

and

$$
S=\left\{\left[\begin{array}{c}
n \\
k \\
\log |\lambda|
\end{array}\right]: n, k \geq 0, \lambda \in \mathbb{C}\right\}
$$

$$
T=\left[\begin{array}{ccc}
0 & 0 & 1 \\
\log \left|a_{2}\right| & \log \left|b_{2}\right| & 1 \\
\log \left|a_{3}\right| & \log \left|b_{3}\right| & 1
\end{array}\right]
$$

then $T$ is a linear transformation from $\mathbb{R}^{3}$ to $\mathbb{R}^{3}$ such that $T(S)$ is dense in $\mathbb{R}^{3}$ thanks to $\bar{G}=\mathbb{R}^{3}$, hence $T$ is onto which implies that $T$ is invertible. Therefore, $S=T^{-1}(T(S))$ must be dense in $\mathbb{R}^{3}$ which is impossible.

To prove the main result of this section, we need the following lemmas.

Lemma 1. For $n>1$ let $x_{1}, x_{2}, \ldots, x_{n-1}$ and $y_{1}, y_{2}, \ldots, y_{n-1}$ be complex numbers. If

$$
M_{n}=\left[\begin{array}{cccccc}
1 & 1 & 1 & 1 & \cdots & 1 \\
x_{1} & y_{1} & 1 & 1 & \cdots & 1 \\
x_{2} & 1 & y_{2} & 1 & \cdots & 1 \\
\vdots & & & & & \\
& & & & \ddots & \\
x_{n-1} & 1 & 1 & 1 & \cdots & y_{n-1}
\end{array}\right]_{n \times n}
$$

is invertible, then the solution of the equation $M_{n} Z=\left[\begin{array}{lllll}1 & 1 & \cdots & 1\end{array}\right]^{T}$, called $Z_{n}=\left[\begin{array}{llll}z_{n}^{1} & z_{n}^{2} & \cdots & z_{n}^{n}\end{array}\right]^{T}$, satisfies the following recursive formula

$$
z_{n}^{i}= \begin{cases}\frac{\left(y_{n-1}-1\right) z_{n-1}^{i}}{\left(y_{n-1}-1\right)+\left(1-x_{n-1}\right) z_{n-1}^{1}}, & 1 \leq i \leq n-1 \\ \frac{\left(1-x_{n-1}\right) z_{n-1}^{1}}{\left(y_{n-1}-1\right)+\left(1-x_{n-1}\right) z_{n-1}^{1}}, & i=n .\end{cases}
$$

Proof. By Crammer's rule we have $z_{n}^{i}=\frac{\operatorname{det} M_{n}^{i}}{\operatorname{det} M_{n}}$, where $M_{n}^{i}$ is the matrix obtained from $M_{n}$ by replacing its $i^{\text {th }}$ column by [ $\left.\begin{array}{lllll}1 & 1 & \cdots & 1\end{array}\right]^{T}$. Expand$\operatorname{ing} \operatorname{det} M_{n}$ and $\operatorname{det} M_{n}^{i}$ along their $n^{t h}$ rows, we can obtain the following recursive formulae:

$$
\begin{aligned}
& \operatorname{det} M_{n}=\left(y_{n-1}-x_{n-1} z_{n-1}^{1}-\sum_{k=2}^{n-1} z_{n-1}^{k}\right) \operatorname{det} M_{n-1}, \\
& \operatorname{det} M_{n}^{i}=\left\{\begin{array}{lc}
\left(y_{n-1}-1\right) \operatorname{det} M_{n-1}^{i}, & 1 \leq i \leq n-1 \\
\left(1-x_{n-1} z_{n-1}^{1}-\sum_{k=2}^{n-1} z_{n-1}^{k}\right) \operatorname{det} M_{n-1}, & i=n .
\end{array}\right.
\end{aligned}
$$

Now an easy computation will give the conclusion; just note that $\sum_{i=1}^{n-1} z_{n-1}^{i}=$ 1. 
Lemma 2. Suppose that $p \geq 3$ is an integer, $t_{1}, \ldots, t_{p-1}$ are real numbers and $f(p)=2-p+\sum_{i=1}^{p-1} t_{i}$. Then the matrix

$$
M_{p}=\left[\begin{array}{ccccc}
1 & 1 & 1 & \cdots & 1 \\
t_{1} & b & 1 & \cdots & 1 \\
t_{2} & 1 & b & \cdots & 1 \\
\vdots & \vdots & & \ddots & \vdots \\
t_{p-1} & 1 & 1 & \cdots & b
\end{array}\right]_{p \times p}
$$

is invertible if and only if $b \notin\{1, f(p)\}$.

Proof. Putting $m_{p}=\operatorname{det} M_{p}$ and writing the expansion of $m_{p}$ via the first column of $M_{p}$, we have $m_{p}=b_{p-1}-c_{p-1} \sum_{i=1}^{p-1} t_{i}$ where

$$
b_{p-1}=\operatorname{det}\left[\begin{array}{ccccc}
b & 1 & 1 & \cdots & 1 \\
1 & b & 1 & \cdots & 1 \\
1 & 1 & b & \cdots & 1 \\
\vdots & \vdots & & \ddots & \vdots \\
1 & 1 & 1 & \cdots & b
\end{array}\right]_{(p-1) \times(p-1)}
$$

and

$$
c_{p-1}=\operatorname{det}\left[\begin{array}{ccccc}
1 & 1 & 1 & \cdots & 1 \\
1 & b & 1 & \cdots & 1 \\
1 & 1 & b & \cdots & 1 \\
\vdots & \vdots & & \ddots & \vdots \\
1 & 1 & 1 & \cdots & b
\end{array}\right]_{(p-1) \times(p-1)}
$$

Multiplying the first row of the matrix in $c_{p-1}$ by -1 and adding it to all other rows, we get $c_{p-1}=(b-1)^{p-2}$. On the other hand, evaluating $b_{p-1}$ by using the first column of the relevant matrix, we find the recursive formula $b_{p-1}=b \cdot b_{p-2}-(p-2) c_{p-2}=b \cdot b_{p-2}-(p-2)(b-1)^{p-3}$. Now, an easy use of the mathematical induction implies that $b_{p-1}=(b-1)^{p-2}(b+p-2)$ and hence we have

$$
m_{p}=(b-1)^{p-2}(b-f(p)) .
$$

Thus, the proof is completed.

In the sequel, some comments are in order. Let $f:[0,1] \rightarrow[0,1]$ be defined by $f(x)=\operatorname{frac}(10 x)$ where $\operatorname{frac}(x)$ denotes the fractional part of real number $x$. For each fixed $n \in \mathbb{N}$, let $F_{2 n}:[0,1]^{2 n} \longrightarrow[0,1]^{2 n}$ be defined by $F_{2 n}\left(x_{1}, x_{2}, \ldots, x_{2 n}\right)=\left(f\left(x_{1}\right), f\left(x_{2}\right), \ldots, f\left(x_{2 n}\right)\right)$. By Proposition 3.1 of [4], there exists $\left(x_{1}, x_{2}, \ldots, x_{2 n}\right) \in[0,1]^{2 n}$ which has a dense orbit under $F_{2 n}$. Let

$$
\triangle^{n}=\left\{\mathbf{x} \in[0,1]^{2 n}: \overline{\operatorname{orb}\left(F_{2 n}, \mathbf{x}\right)}=[0,1]^{2 n}\right\}
$$


and

$$
\triangle_{\frac{1}{2}}^{n}=\left\{\left[\begin{array}{c}
\exp \left(\alpha q_{1} x_{1}+2 \pi i x_{2}\right) \\
\exp \left(\alpha q_{2} x_{3}+2 \pi i x_{4}\right) \\
\vdots \\
\exp \left(\alpha q_{n} x_{2 n-1}+2 \pi i x_{2 n}\right)
\end{array}\right]:\left(x_{1}, x_{2}, \ldots, x_{2 n}\right) \in \triangle^{n}, q \in \mathbb{N}, \alpha=\ln 2\right\} .
$$

By Corollary 3.5 of [4], for every $\left(a_{1}, a_{2}, \ldots, a_{n}\right) \in \triangle_{\frac{1}{2}}^{n},\left|a_{i}\right|>1(i=1,2, \ldots, n)$. Moreover, if $A_{n}$ and $B_{n k}(1 \leq k \leq n)$ are diagonal matrices with, respectively, the diagonals $\left(a_{1}, a_{2}, \ldots, a_{n}\right)$ and $\left(b_{1 k}, b_{2 k}, \ldots, b_{n k}\right)$ where $b_{k k}=\frac{1}{2}$ and $b_{i k}=1$ $(i \neq k)$, then the $(n+1)$-tuple $\left(A_{n}, B_{n 1}, \ldots, B_{n n}\right)$ is hypercyclic on $\mathbb{C}^{n}$ with the hypercyclic vector $\nu=\left[\begin{array}{llll}1 & 1 & \cdots & 1\end{array}\right]^{T}$. Hence the set

$$
E=\left\{\left[\begin{array}{c}
2^{-k_{1}} a_{1}{ }^{m} \\
2^{-k_{2}} a_{2}{ }^{m} \\
\vdots \\
2^{-k_{n}} a_{n}^{m}
\end{array}\right]: m \geq 0, k_{i} \geq 0, i=1,2, \ldots, n\right\}
$$

is dense in $\mathbb{C}^{n}$.

Theorem 2. There is a supercyclic spherical isometric $p$-tuple on $\mathbb{C}^{p}, p \geq 1$.

Proof. The case $p=1$ is obvious. Now, let $p=n+1 \geq 2$ and consider the $p$-tuple $\left(A_{n}^{\prime}, B_{n 1}^{\prime}, \ldots, B_{n n}^{\prime}\right)$ of diagonal matrices on $\mathbb{C}^{p}$, where $A_{n}^{\prime}$ and $B_{n k}^{\prime}(1 \leq$ $k \leq n)$ have, respectively, the diagonals $\left(1, a_{1}, a_{2}, \ldots, a_{n}\right)$ and $\left(1, b_{1 k}, \ldots, b_{n k}\right)$. Also, let $\nu^{\prime}=\left[\begin{array}{llll}1 & 1 & \cdots & 1\end{array}\right]^{T}$. Then the density of $E$ in $\mathbb{C}^{n}$ implies that

$$
\left\{\lambda\left(A_{n}^{\prime}\right)^{k_{1}}\left(B_{n 1}^{\prime}\right)^{k_{2}} \cdots\left(B_{n n}^{\prime}\right)^{k_{p}} \nu^{\prime}: \lambda \in \mathbb{C}, k_{i} \geq 0, i=1,2, \ldots, n+1\right\}
$$

is dense in $G=\mathbb{C}^{p}-\left\{\left(0, \lambda_{1}, \ldots, \lambda_{n}\right): \lambda_{i} \in \mathbb{C}, i=1,2, \ldots, n\right\}$. But $G$ is also dense in $\mathbb{C}^{p}$, so we conclude that the vector $\nu^{\prime}$ is supercyclic for the $p$-tuple $\left(A_{n}^{\prime}, B_{n 1}^{\prime}, \ldots, B_{n n}^{\prime}\right)$. We claim that there are scalars $\alpha_{1}, \alpha_{2}, \ldots, \alpha_{p}$ such that the $p$-tuple $\left(\alpha_{1} A_{n}^{\prime}, \alpha_{2} B_{n 1}^{\prime}, \ldots, \alpha_{p} B_{n n}^{\prime}\right)$ is a supercyclic spherical isometry on $\mathbb{C}^{p}$. For $p=2$ if $\left|a_{1}\right|>1$ and we put

$$
\alpha_{1}=\sqrt{\frac{3}{4\left|a_{1}\right|^{2}-1}}, \alpha_{2}=\sqrt{\frac{4\left(\left|a_{1}\right|^{2}-1\right)}{4\left|a_{1}\right|^{2}-1}}, A_{1}^{\prime}=\left[\begin{array}{cc}
1 & 0 \\
0 & a_{1}
\end{array}\right], B_{11}^{\prime}=\left[\begin{array}{cc}
1 & 0 \\
0 & \frac{1}{2}
\end{array}\right] \text {, }
$$

then $\left(\alpha_{1} A_{1}^{\prime}, \alpha_{2} B_{11}^{\prime}\right)$ is a spherical isometric 2-tuple on $\mathbb{C}^{2}$. Let $p=n+1 \geq$ 3. The $p$-tuple $\left(\alpha_{1} A_{n}^{\prime}, \alpha_{2} B_{n 1}^{\prime}, \ldots, \alpha_{p} B_{n n}^{\prime}\right)$ is spherical isometry if and only if there is a solution $Z_{p}=\left[\begin{array}{lll}z_{p}^{1} & \cdots & z_{p}^{p}\end{array}\right]^{T}$ for the equation $M_{p} Z=$ $\left[\begin{array}{llll}1 & 1 & \cdots & 1\end{array}\right]^{T}$, where

$$
M_{p}=\left[\begin{array}{ccccc}
1 & 1 & 1 & \cdots & 1 \\
\left|a_{1}\right|^{2} & \frac{1}{4} & 1 & \cdots & 1 \\
\left|a_{2}\right|^{2} & 1 & \frac{1}{4} & \cdots & 1 \\
\vdots & \vdots & & \ddots & \vdots \\
\left|a_{p-1}\right|^{2} & 1 & 1 & \cdots & \frac{1}{4}
\end{array}\right]
$$


and $z_{p}^{i}>0$ for $i=1,2, \ldots, p$. Note that by Lemma 2, $M_{p}$ is invertible for all $p \geq 3$. Suppose that $\left(a_{1}, a_{2}, \ldots, a_{p}\right) \in \triangle_{\frac{1}{2}}^{p}$ is the point corresponding to $\left(x_{1}, x_{2}, \ldots, x_{2 p}\right) \in \triangle^{p}$. Since $\left(x_{1}, x_{2}, \ldots, x_{2 p}\right)$ has a dense orbit under $F_{2 p}$, it is easy to see that $\left(x_{1}, x_{2}, \ldots, x_{2 p-2}\right)$ has a dense orbit under $F_{2 p-2}$ and clearly $\left(a_{1}, a_{2}, \ldots, a_{p-1}\right) \in \triangle_{\frac{1}{2}}^{p-1}$ is the point corresponding to $\left(x_{1}, x_{2}, \ldots, x_{2 p-2}\right) \in$ $\triangle^{p-1}$. Therefore, by Lemma 1 , the existence of $z_{p}^{i}>0, i=1,2, \ldots, p$, such that $M_{p} Z_{p}=\left[\begin{array}{lllll}1 & 1 & \cdots & 1\end{array}\right]^{T}$, depends on the existence of $z_{p-1}^{i}>0$, $i=1,2, \ldots, p-1$, such that $M_{p-1} Z_{p-1}=\left[\begin{array}{lllll}1 & 1 & \ldots & 1\end{array}\right]^{T}$ and obviously, this backward process can be continued to conclude finally that the existence of $z_{p}^{i}>0$, for $i=1,2, \ldots, p$ depends on the existence of $z_{2}^{i}>0, i=1,2$, such that $M_{2} Z_{2}=\left[\begin{array}{l}1 \\ 1\end{array}\right]$, which is proved.

We finish this note by giving the following result which is a consequence of Example 2.11 and Corollary 4.2 of [4].

Proposition 1. There is no supercyclic spherical or toral isometry on an infinite-dimensional Hilbert space.

Proof. We prove the assertion for spherical isometries. The same argument gives the proof for toral isometries. Suppose that $H$ is an infinite-dimensional Hilbert space and $\left(A_{1}, A_{2}, \ldots, A_{n}\right)$ is a supercyclic spherical isometry on $H$. By a result of [1], the tuple $\left(A_{1}, A_{2}, \ldots, A_{n}\right)$ is subnormal; that is, there is a Hilbert space $K$ containing $H$ and a commuting tuple $\left(S_{1}, S_{2}, \ldots, S_{n}\right)$ of normal operators on $K$ such that $A_{i}=\left.S_{i}\right|_{H}$ for $i=1,2, \ldots, n$. Let $\theta$ be an irrational multiple of $\pi$ and $a, b$ be two relatively prime integers greater than one. Moreover, suppose that $I$ is the identity operator on $H$. By Corollary 4.2 of [4] the set $\left\{\frac{a^{k_{1}} e^{i k_{2} \theta}}{b^{k_{3}}}: k_{1}, k_{2}, k_{3} \geq 0\right\}$ is dense in $\mathbb{C}$; thus, the supercyclicity of $\left(A_{1}, A_{2}, \ldots, A_{n}\right)$ implies the hypercyclicity of $(n+3)$-tuple $\left(a I, \frac{1}{b} I, e^{i \theta} I, A_{1}, A_{2}, \ldots, A_{n}\right)$, but this is a subnormal tuple and, by Corollary 3.9 of [4], can not be hypercyclic.

Remark 1. In fact the proof of Proposition 1 shows that on an infinite-dimensional Hilbert space. There is no supercyclic n-tuple of subnormal operators with commuting normal extensions.

Acknowledgments. This research was in part supported by a grant from Shiraz University Research Council.

\section{References}

[1] A. Athavale, On the intertwining of joint isometries, J. Operator Theory 23 (1990), no. $2,339-350$.

[2] M. Faghih Ahmadi and K. Hedayatian, Hypercyclicity and supercyclicity of m-isometric operators, Rocky Mountain J. Math. 42 (2012), no. 1, 15-23.

[3] N. S. Feldman, Hypercyclic pairs of coanalytic Toeplitz operators, Integral Equations Operator Theory $\mathbf{5 8}$ (2007), no. 2, 153-173. 
[4] - Hypercyclic tuples of operators and somewhere dense orbits, J. Math. Anal. Appl. 346 (2008), no. 1, 82-98.

[5] H. M. Hilden and L. J. Wallen, Some cyclic and non-cyclic vectors of certain operators, Indiana Univ. Math. J. 23 (1973/74), 557-565.

[6] R. Soltani, K. Hedayatian, and B. Khani Robati, On supercyclicity of tuples of operators, to appear in the Bull. Malays. Math. Sci. Soc.

[7] R. Soltani, B. Khani Robati, and K. Hedayatian, Hypercyclic tuples of the adjoint of the weighted composition operators, Turkish J. Math. 36 (2012), no. 3, 452-462.

Mohammad Ansari

Department of Mathematics

COLlege of SCIENCES

SHIRAZ UNIVERSITY

SHIRAZ 71454, IRAN

E-mail address: m_ansari@shirazu.ac.ir

Karim Hedayatian

Department of Mathematics

College of Sciences

Shiraz University

SHIRAZ 71454, IRAN

E-mail address: hedayati@shirazu.ac.ir

Bahram Khani-Robati

Department of Mathematics

College of Sciences

SHIRAZ UNIVERSITY

SHIRAZ 71454, IraN

E-mail address: bkhani@shirazu.ac.ir

Abbas Moradi

Department of Mathematics

College of Sciences

SHIRAZ UNIVERSITY

SHIRAZ 71454, IRAN

E-mail address: amoradi@shirazu.ac.ir 